\title{
Notes on Mathematical Problems on the Dynamics of Dispersed Particles interacting through a Fluid
}

\author{
P.E. Jabin $\left({ }^{*}\right)$ and B. Perthame $\left({ }^{*}\right)\left({ }^{* *}\right)$ \\ $\left({ }^{*}\right)$ Ecole Normale Supérieure, DMI \\ 45, rue d'Ulm \\ 75230 Paris Cedex 05, France \\ $\left({ }^{* *}\right)$ INRIA-Rocquencourt, Projet M3N \\ BP 105 \\ 78153 Le Chesnay Cedex, France
}

\section{Introduction}

In this Chapter, we present some mathematical problems related to the dynamics of particles interacting through a fluid. We are interested in the dilute cases. We mean the cases where a transport Partial Differential Equations in the phase space can be expected for the particles density. In order to derive these transport equations explicitely, some assumptions on the fluid dynamics are necessary. They limit the validity of the model but still represents many possible applications. Namely we assume that the fluid dynamics can be reduced to two simple situations. The first situation is the simple case of a potential flow (perfect incompressible and irrotational flow). This is relevant to describe for instance the motion of bubbles in water (see G.K. Batchelor [2]) and focuses mainly on the added mass effect which means that to accelarate bubbles requires to accelerate some part of the water too. The second situation is the more standard case of particles in a Stokes flow, for which the domains of application are suspensions or sedimendation. 
The case of a potential flow around the particles, leads to a difficulty in establishing the equation for the particle density. A mathematical formalism was developed by G. Russo and P. Smereka [26] which we will present here, in the improved version of H. Herrero, B. Lucquin and B. Perthame [19]. We will recall here how one can derive, from the interacting system of particles, a Vlasov type of equation for the particle density in the phase space $g(t, x, p)$, here $t \geq 0$ is the time, $x \in \mathbb{R}^{3}$ represents the space position and $p \in \mathbb{R}^{3}$ represents the total impulsion of particles (dual of the velocity in the Lagrangian - Hamiltonian duality). This equation is

$$
\begin{gathered}
\frac{\partial}{\partial t} g+\operatorname{grad}_{p} H \cdot \operatorname{grad}_{x} g-\operatorname{grad}_{x} H \cdot \operatorname{grad}_{p} g=0, \\
H(t, x, p)=\frac{1}{2}|p+\Phi(t, x)|^{2}, \\
\Phi(t, x)=\lambda B *(\mathbf{P}+\rho \Phi)(t, .) .
\end{gathered}
$$

Here $B=B(x)$ is a given $3 \times 3$ matrix, $\lambda$ is the kinetic parameter (relating the radius of the particles to the densities of the particles and of the fluid) and the macroscopic density and implusion $\rho, \mathbf{P}$ are defined by

$$
\begin{aligned}
\rho(t, x) & =\int_{\mathbb{R}^{3}} g(t, x, p) d p, \\
\mathbf{P}(t, x) & =\int_{\mathbb{R}^{3}} p g(t, x, p) d p .
\end{aligned}
$$

The difficulty to establish this equation, comes from the Lagrangian aspect of the natural dynamics for the particles. It turns out that the Hamiltonian variables are better adapted to mathematical manipulations and to mechanical interpretation (notice that the Hamiltonian variable is just the total impulsion of particles). But the derivation of the mean field equation (1.1)(1.3) for the particles density is easier in Lagrangian variables. Then, one issue is to understand how to define, in the kinetic P.D.E., the Lagrangian and Hamiltonian variables (and to understand also change of variables).

The second situation consists in considering a Stokes flow around the particles. It leads to quite different mathematical issues. In order to establish equations for the particle density one can follow the same derivation as before. From the full dynamics of particles - $\mathrm{N}$ body interaction - a first (and restrictive) assumption is to make a dipole approximation for the fluid equation. This reduces the dynamics to two-body interactions and thus allows 
to settle the kinetic equation for the particle density $f(t, x, v)$, here $v$ is the velocity of the particle. One obtains a Vlasov type equation.

$$
\begin{gathered}
\frac{\partial}{\partial t} f+v \cdot \operatorname{grad}_{x} f+\lambda \operatorname{div}_{v}\left(\left(\kappa g+\mu A \star_{x} j-v\right) f\right)=0, \\
j(t, x)=\int_{\mathbb{R}^{3}} v f(t, x, v) d v .
\end{gathered}
$$

The matrix $A(x)$ is now related to the Stokes Equation, as well as $B$, in the potential case, is related to the Laplace Equation. Also, $g$ denotes the gravity vector, $\lambda$ the kinetic parameter and $\mu=\frac{3}{4} N a$, with $N$ the number of particles, $a$ their radius. Even though there is no mathematical difficulty in establishing this system, several mathematical questions arise concerning, for instance, various asymptotic behaviors (large time behavior cf [21], $\lambda$ vanishing...etc) They arise because the friction term plays a major role in the particles dynamics for a Stokes flow. A particularly interesting situation is the limit $\lambda \rightarrow \infty$. It gives an example of a macroscopic limit which is not obtained by the collisional process, but by a strong force term. In the case at hand, it is proved in P.E. Jabin [22] that the macroscopic limit gives rise to the equation

$$
\begin{aligned}
& \frac{\partial}{\partial t} \rho+\operatorname{div}(\rho u)=0, \\
& \mu A \star_{x}(\rho u)-u=g .
\end{aligned}
$$

The topic of these notes represent particular examples of a very active field of fluid mechanics where kinetic physics plays a fundamental role. Usually it is used in the derivation of models for particular situations, but also of effective equations for the motion. In no way we can give a complete account of the literature in this domain and we prefer to refer to some general works. Concerning bubbly-potential flows, the paper by Y. Yurkovetsky and J.F. Brady [32] contains numerous recent references as well as considerations on statistical physics aspects of the model and the effect of collisisons. For this effect, see also G. Russo and P. Smereka [27], J.F. Bourgat et al [6]. The derivation of pde models and the use of kinetic description is a rather recent subject, confer H.F. Bulthuis, A. Prosperetti and A.S. Sangani [7], A.S. Sangani and A.K. Didwana [28], P. Smereka [30] and the references therein. On the other hand, the dynamics of particles in a Stokes flow have lead to very numerous works. Let us quote some of them : G.K. Batchelor and C.S. Wen 
[8], F. Feuillebois [12], E.J. Hinch [16], R. Herczynski and I. Pienkowska [20] and the book by J. Happel and H. Brenner [17]. Other regimes have also been studied and lead to mathematical models which have been analyzed for instance by K. Hamdache [18] for the case of a more general incompressible flow (and small particles), by D. Benedetto, E. Caglioti and M. Pulvirenti [3] for granular flow. Complex numerical simulations have been performed by B. Maury and R. Glowinski [25], R. Glowinski, T.W. Pan and J. Périaux [15], for high concentrations of particles (see also the references therein).

The outline of this Chapter is as follows. The next two sections are devoted to the case of a potential flow ; in section 2 we derive the model dynamical system and section 3 is devoted to the mean field equation. In the fourth section, we derive the dynamical system for the case of Stokes flow. The macroscopic limit is explained in Section 5. Some numerical tests for the potential flow case are presented in the Appendix.

The sections are largely independant of each other. Except some notations which are refered to in the text, they can be read independently.

\section{Dynamics of Balls in a Potential Flow}

In this Section, we consider the dynamics of $N$ balls of radius $a$, interacting through a potential fluid. The motion of each ball modifies the global flow and thus produces a force on the other balls. Even though we consider the very simplified situation of the dipole approximation of a potential flow, the result is a complex dynamics. Here, we describe (under the assumption of diluted particles), the limiting behavior, as $N \rightarrow \infty$ and $a$ vanishes, of the particles density. As we will see in Section II, as long as collisions between particles are neglected and a specific relation holds between $a$ and $N$, this leads to the equation (1.1)-(1.3) for the distribution of particles in the phase space (time, space and total impulsion).

Our notations are as follows. We consider $N$ particles which centers are denoted $X_{i}(t)$, they move with velocities $V_{i}(t)$. Here, $t$ denotes the time and $1 \leq i \leq N$. These particles are balls of radius $a$, centered at $X_{i}(t)$, they are denoted $B_{i}(t)$. The inward normal on the sphere $\partial B_{i}(t)$ will be denoted by $n_{i}(x)$. We also denote by $\rho_{f}$ the fluid density and by $\rho_{p}$ the particle density, their mass is thus $m_{p}=\frac{4}{3} \pi a^{3} \rho_{p}$, another remarquable quantity which arises 
later is the virtual mass of the fluid $m_{v}=\frac{2}{3} \pi a^{3} \rho_{f}$. As we will see, there is a fundamental number which decides of the validity of a dilute regime, it is given by

$$
\lambda=6 \pi N a^{3} \frac{\rho_{f}}{\rho_{f}+2 \rho_{p}}
$$

This ratio has to be kept constant in the limit $N \rightarrow \infty$. Finally, we use calgraphic letters for the $3 N$ dimensional vectors (or matrices acting on these vectors). For instance $\mathcal{X}_{N}=\left(X_{1}, \ldots, X_{N}\right)$.

\section{1 the full dynamics}

The fluid around the particles is assumed to be given by the potential equations. In other words, the fluid velocity $v(t, x)$ is obtained as

$$
v(t, x)=\nabla \phi(t, x),
$$

where the potential $\phi$ is just given by

$$
\left\{\begin{array}{l}
\Delta \phi(t, x)=0 \text { in } \mathbb{R}^{3}-\bigcup_{\mathrm{i}=1}^{\mathrm{N}} \mathrm{B}_{\mathrm{i}}(\mathrm{t}), \\
\frac{\partial \phi}{\partial n_{i}}=V_{i}(t) \cdot n_{i} \text { on } \partial B_{i}(t) .
\end{array}\right.
$$

We implicity consider that the fluid is at rest at infinity, i.e. $v$ vanishes at infinity. From this potential $\phi$, we can compute the pressure thanks to the Bernouilli relation

$$
p(t, x)=-\rho_{f}\left(\frac{\partial \phi}{\partial t}+\frac{|\nabla \phi|^{2}}{2}\right) .
$$

This makes $v(t, x)$ a solution of the incompressible Euler Equations. The dynamics of the particles is therefore defined by the fundamental principle of dynamics,

$$
\left\{\begin{array}{c}
\dot{X}_{i}(t)=V_{i}(t) \\
m_{p} \dot{V}_{i}(t)=F_{i}(t)=\int_{\partial B_{i}(t)} p(t, x) n_{i} d S .
\end{array}\right.
$$

Let us point out that the force $F_{i}$ depends upon all the positions $X_{i}(t)$ and velocities $V_{i}(t)$ of the particles, and also of their derivatives. Especially $F_{i}$ depends upon $\frac{d}{d t} V_{i}(t)$. This shows that this dynamics is rather complex and is not explicitely solvable by the Cauchy-Lipschitz Theorem. But it has a Lagrangian structure which gives a way to study it. Indeed, we have 
Theorem 2.1 There is a $N \times N$ symmetric positive definite matrix $A_{i j}\left(\mathcal{X}_{N}\right)$, such that the system (2.5) admits the Lagrangian

$$
\mathcal{L}_{N}=\frac{1}{2} \sum_{i, j=1}^{N} V_{i}^{t} A_{i j} V_{j} .
$$

In other word it can be written

$$
\left\{\begin{array}{l}
\frac{d}{d t} X_{i}(t)=V_{i}(t) \\
\frac{d}{d t} \frac{\partial \mathcal{L}_{N}(t)}{\partial V_{i}}=\frac{\partial \mathcal{L}_{N}(t)}{\partial X_{i}} .
\end{array}\right.
$$

The $3 N \times 3 N$ matrix $A_{i j}$ is usually called the added mass matrix. One of the difficulties is that each term $A_{i j}$ depends on the full vector $\mathcal{X}_{N}$.

Remark 2.2 (i) We have set

$$
\mathcal{L}_{N}(t)=\mathcal{L}_{N}\left(X_{1}(t), \ldots, X_{N}(t) ; V_{1}(t), \ldots, V_{N}(t)\right)
$$

(ii) The dynamics is not well defined. Indeed, $\phi$ is only defined as long as the distance between two particles is larger that $2 a$. When they touch, a collision process should be defined. To avoid these physical considerations we will in fact extend the definition of $A_{i j}$ such that it vanishes when two particles are too close (distance less than a say).

Proof of Theorem 2.1. See [30], [26], [19]. We would like however to mention an intermediary step. We define the Kelvin impulsion by

$$
I_{i}(t)=\rho_{f} \int_{\partial B_{i}(t)} \phi(t, x) n_{i} d S
$$

And the total impulsion is then defined as

$$
P_{i}(t)=m_{p} V_{i}(t)+I_{i}(t) .
$$

Then, one computes

$$
\dot{I}_{i}(t)=\rho_{f} \int_{\partial B_{i}(t)}\left[\frac{\partial}{\partial t} \phi(t, x)+\dot{X}_{i}(t) \cdot \nabla \phi\right] n_{i} d S .
$$


Therefore, combining this equality with (2.4), (2.5), the force field is given by

$$
F_{i}(t)=-\dot{I}_{i}(t)-\rho_{f} \int_{\partial B_{i}(t)} \nabla \phi\left[\nabla \phi-V_{i}(t)\right] n_{i} d S .
$$

This gives the formula

$$
\frac{\partial \mathcal{L}_{N}}{\partial V_{i}}=m_{p} V_{i}+\rho_{f} \int_{\partial B_{i}(t)} \phi(t, x) n_{i} d x
$$

And one can easily check that this expression is linear in $\left(V_{i}\right)_{1 \leq i \leq N}$,

$$
\frac{\partial \mathcal{L}_{N}}{\partial V_{i}}=\sum_{j=1}^{N} A_{i j}\left(\mathcal{X}_{N}\right) V_{j},
$$

from which the expression (2.6) follows.

\section{2 the method of reflections}

The solution $\phi$ to the potential equation (2.3) is very difficult to use because it depends in a complex way on the positions of the particles. However, a general and simple formal method allows to build the solution as the sum of a series expansion, and therefore to find approximations. This is the method of reflections, also called the method of images. It is based on a systematic reduction to a single ball which allows to represent the potential as

$$
\phi(t, x)=\sum_{n=1}^{\infty} \sum_{i=1}^{N} \phi_{i}^{(n)}(t, x) .
$$

The construction of $\phi_{i}^{(n)}(t, x)$ is by recursion. We first set

$$
\left\{\begin{array}{l}
\Delta \phi_{i}^{(1)}(t, x)=0 \text { in } \mathbb{R}^{3}-\mathrm{B}_{\mathrm{i}}(\mathrm{t}), \\
\frac{\partial \phi_{i}^{(1)}}{\partial n_{i}}=V_{i}(t) \cdot n_{i} \text { on } \partial B_{i}(t) .
\end{array}\right.
$$

Considering $\phi^{(1)}=\sum_{i=1}^{N} \phi_{i}^{(1)}$, we see that we realize the Laplace equation in (2.3), but we miss the boundary condition. In a second step, we therefore correct this boundary condition setting by recursion

$$
\left\{\begin{array}{l}
\Delta \phi_{i}^{(n)}(t, x)=0 \text { in } \mathbb{R}^{3}-\mathrm{B}_{\mathrm{i}}(\mathrm{t}), \\
\frac{\partial \phi_{i}^{(n)}}{\partial n_{i}}=-\sum_{j \neq i} \frac{\partial \phi_{j}^{(n-1)}}{\partial n_{i}} \text { on } \partial B_{i}(t) .
\end{array}\right.
$$


Adding these equations, we immediately see that the potential (2.13) formally satisfies the equation (2.3). However it is not sure that the series converges, and this certainly requires a smallness assumption relating the balls size $a$, the distance $d_{i j}$ between them, and the number of balls $N$.

Remark 2.3 (Case of one ball) The solution to the equation on $\phi_{i}^{(1)}$ is very simple. It corresponds to the formula for a single ball

$$
\phi_{i}^{(1)}(x)=-\frac{a^{3}}{2} V_{i} \cdot \frac{x-X_{i}}{\left|x-X_{i}\right|^{3}} .
$$

It gives

$$
\int_{\partial B_{i}} \phi_{i}^{(1)} n_{i} d S=-\frac{2 \pi}{3} a^{3} V_{i} .
$$

And, by a simple expansion, we also find

$$
\int_{\partial B_{i}} \phi_{j}^{(1)} n_{i} d S=-\frac{4 \pi}{3} a^{3} \tilde{W}_{j}+a^{3} 0\left(\left(a / d_{i j}\right)^{5}\right),
$$

with

$$
\begin{gathered}
\tilde{W}_{j}=\frac{a^{3}}{2}\left[\frac{V_{j}}{\left|X_{j}-X_{i}\right|^{3}}-3 V_{j} \cdot\left(X_{j}-X_{i}\right) \frac{X_{j}-X_{i}}{\left|X_{j}-X_{i}\right|^{5}}\right], \\
d_{i j}=\left|X_{I}-X_{j}\right| .
\end{gathered}
$$

\section{3 the dipole approximation}

Since we are in the situation where $a$ is vanishing, and in a dilute regime, the above construction makes sense. Also, going one step further, it is natural to truncate the expansion. It is not possible to just consider the dominant term acting on $B_{j}$, i.e. $\phi_{j}^{(1)}$ because, by d'Alembert paradoxe, it creates no force on the ball $B_{j}$. One step further again, we consider the first 'image' $\sum_{i=1}^{N} \phi_{i}^{(2)}$, and also one can still get the same order of truncation in using appropriate asymptotic expansions in $\left(a / d_{i j}\right)$. We describe the computations in this subsection.

From the computation of forces created by a single bubble in the Remark 2.3 , we obtain the implusion created by $\phi^{(1)}$,

$$
\int_{\partial B_{i}(t)} \phi^{(1)}(t, x) n_{i} d S=-\frac{2 \pi}{3} a^{3} V_{i}-\frac{4 \pi}{3} a^{3} \tilde{V}_{i}(t)+a^{3} O\left(\left(\frac{a}{d}\right)^{5}\right),
$$


where

$$
\begin{aligned}
\tilde{V}_{i} & =\frac{a^{3}}{2} \sum_{j \neq i}\left[\frac{V_{j}}{\left|X_{j}-X_{i}\right|^{3}}-3 V_{j} \cdot\left(X_{j}-X_{i}\right) \frac{X_{j}-X_{i}}{\left|X_{j}-X_{i}\right|^{5}}\right] \\
& =\frac{a^{3}}{2} \sum_{j \neq i} V_{j} \cdot D^{2}\left(\frac{1}{\left|X_{j}-X_{i}\right|}\right),
\end{aligned}
$$

(we mean $D^{2} \frac{1}{|x|}$ evaluated at $X_{i}-X_{j}$ ). Then, in the series expansion (2.13) there is another term of order $(a / d)^{3}$. Namely, the contribution of the term $\phi_{i}^{(2)}$ on the ball $B_{i}$, has the same order. Indeed, for small values of the ratio $a / d_{i j}$, we have,

$$
\frac{\partial}{\partial n_{i}} \phi_{i}^{(2)} \approx \tilde{V}_{i} \cdot n_{i} \quad \text { on } \partial B_{i}(t)
$$

and thus, using again the case of a single bubble,

$$
\phi_{i}^{(2)}(x) \approx-\frac{a^{3}}{2} \tilde{V}_{i} \cdot \frac{x-X_{i}}{\left|x-X_{i}\right|^{3}} .
$$

Computing its contribution to the Lagrangian, we finally end up with the following formula for Kelvin's impulsion (2.9),

$$
I_{i}(t)=\frac{2 \pi}{3} \pi a^{3} \rho_{f}\left[V_{i}(t)+3 \tilde{V}_{i}(t)+O\left(\left(\frac{a}{d}\right)^{4}\right)\right] .
$$

Hence the total impulsion (2.10) is given by

$$
P_{i}(t)=\left(m_{p}+m_{v}\right) V_{i}(t)+3 m_{v} \tilde{V}_{i}(t)+m_{v} O\left(\left(\frac{a}{d}\right)^{4}\right) .
$$

Here we have denoted by $m_{v}$ the virtual mass

$$
m_{v}=\frac{2 \pi}{3} \pi a^{3} \rho_{f} .
$$

Neglecting the remainder terms gives the final expression

$$
\begin{gathered}
P_{i}=\sum_{j=1}^{N} A_{i j} V_{j}, \\
A_{i i}=\left(m_{p}+m_{v}\right) I d, \quad \text { for } i \neq j . \\
A_{i j}=-\frac{3}{2} m_{v} a^{3} D^{2}\left(\frac{1}{\left|X_{j}(t)-X_{i}(t)\right|}\right) \text {. }
\end{gathered}
$$

Therefore, we obtain, under the dipole approximation, the Lagangian (2.6) defined by the above symmetric added mass matrix which is now simpler because it is two-point additive. Unlike the original case, this matrix is not always invertible. In the next section, we will give some invertibility properties. 


\section{Kinetic theory for the Hamiltonian system of bubbly flows}

For particles moving with the dynamic described in the previous section, the mean field equation for the density in the phase space can be derived either in Lagrangian or in Hamiltonian variables. Nevertheless, as explained in the introduction, the Hamiltonian variables give a nicer structure. In this Section, we consider a more general dynamic for the particles, with the same type of Lagrangian structure, we recall it in Subsection 1. In Subsection 2, we present the Hamiltonian variables, and the kinetic system (1.1)-(1.3) is derived in Subsection 3.

\section{1 the general Lagrangian structure}

From the approximation in Section 1, we are led to study the more general Lagrangian system, for $1 \leq i \leq N$,

$$
\begin{gathered}
\left\{\begin{array}{c}
\frac{d}{d t} X_{i}(t)=V_{i}(t), \\
\frac{d}{d t} \frac{\partial \mathcal{L}_{N}(t)}{\partial V_{i}}=\frac{\partial \mathcal{L}_{N}(t)}{\partial X_{i}},
\end{array}\right. \\
\mathcal{L}_{N}=\frac{1}{2} \sum_{i, j=1}^{N} V_{i}^{t} A_{i j} V_{j} .
\end{gathered}
$$

And we recall the notation (2.8) for $\mathcal{L}_{N}(t)$. The outcome of the result in [19] that we recall here, is that it is possible to give the asymptotic limit as $N \rightarrow \infty$ for quadratic Lagrangian in $V_{i}$, under a particular two-point additivity assumption for the added mass matrix $\mathcal{A}_{N}=\left(A_{i j}\right)_{1 \leq i, j \leq N}$. Since the Lagrangian is defined up to a multiplicative constant, it is consistent with (2.18), to consider $3 \times 3$ matrices of the form

$$
\begin{aligned}
& A_{i i}=I d, \\
& A_{i j}=-\frac{\lambda}{N} B\left(X_{j}-X_{i}\right) \quad \text { for } i \neq j .
\end{aligned}
$$

In the above derivation, the positive parameter $\lambda$ is given by (2.1), but here this explicit relation is useless. Also the $3 \times 3$ matrix $B$ is given by the particular form

$$
\underline{B}(x)=D^{2} \frac{1}{4 \pi|x|},
$$


(recall that the distance between particles is never less than $2 a$ ). But our analysis requires smooth matrices $B$ and we will use the assumption

$$
B \text { is even, } \quad B \text { and } D^{2}(B) \text { are bounded, } B(0)=0 \text {. }
$$

Before proving that for such a Lagrangian system, the limiting behavior as $N \rightarrow \infty$ is given by the Vlasov Equation (1.1)-(1.3), we need to pass to the Hamiltonian variables (at least to prove existence of solutions to (3.1) for instance).

\section{2 the corresponding Hamiltonian structure}

As usual the Hamiltonian structure is defined through the Frenchel dual of the quadratic Lagrangian (at least for $\lambda$ small enough). We therefore set

$$
\mathcal{H}_{N}\left(X_{1}, X_{2}, \ldots, X_{N} ; P_{1}, P_{2}, \ldots, P_{N}\right)=\frac{1}{2} \sum_{i, j=1}^{N} P_{i}^{t}\left(\mathcal{A}_{N}^{-1}\right)_{i j} P_{j},
$$

- a justification of the invertibility of the matrix $\mathcal{A}_{N}=\left(A_{i j}\right)_{1 \leq i, j \leq N}$ is given later. Notice however that the inverse matrix of $\left(A_{i j}\right)_{1 \leq i, j \leq N}$ is not two point additive (its coeficients depend on the full vector $\mathcal{X}_{N}$ ). As usual the change of variables from velocoties $V_{i}$ to impulsions $P_{i}$ is obtained by the formulae

$$
\begin{aligned}
P_{i} & =\frac{\partial \mathcal{L}_{N}}{\partial V_{i}}, \\
V_{i} & =\frac{\partial \mathcal{H}_{N}}{\partial P_{i}},
\end{aligned}
$$

And the Lagrangian system (3.1) is equivalent to the Hamiltonian system (see [1] for instance)

$$
\begin{aligned}
& \dot{X}_{i}(t)=\frac{\partial \mathcal{H}_{N}(t)}{\partial P_{i}}, \\
& \dot{P}_{i}(t)=-\frac{\partial \mathcal{H}_{N}(t)}{\partial X_{i}} .
\end{aligned}
$$

In order to state a precise statement for this equivalence, we need a last notation : $\|B\|_{2}$ denotes the matrix norm of the $3 \times 3$ matrix $B$ induced by the euclidian norm in $\mathbb{R}^{3}$. Then, we have

Theorem 3.1 (see [19]). We assume that

$$
\lambda \sup _{x \in \mathbb{R}^{3}}\|B(x)\|_{2}<1 .
$$

Then, the matrix $\left(A_{i j}\right)_{1 \leq i, j \leq N}$ defined in (3.3) is invertible, the equations (3.1) and (3.8) are equivalent, and they are wellposed. 
The existence of global solutions to the systems (3.1) and (3.8) follows from the Cauchy-Lipschitz Theorem. Indeed, under the assumption (3.5), the Hamiltonian system is Lipschitzian. Also, under the assumption (3.9), the invertibilty of the matrix $A_{i j}$ follows from the convergence (in norm) of the series expansion

$$
\left(A^{-1}\right)_{i j}=I d+\frac{\lambda}{N} B\left(X_{j}-X_{i}\right)+\left(\frac{\lambda}{N}\right)^{2} \sum_{k=1}^{N} B\left(X_{k}-X_{i}\right) B\left(X_{j}-X_{k}\right)+\ldots
$$

\section{3 the mean field equation}

We are now interested in the particles density in the phase space

$$
g_{N}(t, x, p)=\frac{1}{N} \sum_{i=1}^{N} \delta\left(x-X_{i}(t)\right) \otimes \delta\left(p-P_{i}(t)\right) .
$$

The interest of using this formalism is that it allows to pass to the limit as $N \rightarrow \infty$.

Theorem 3.2 ([19]). With the assumption of Theorem 3.1, the Hamiltonian system (3.8) is equivalent to the Vlasov system (1.1)-(1.3) on the measure $g_{N}$ (see the introduction). Also, the equation (1.3) on the vector potential $\Phi$ has a unique solution (being given $\rho$ and $\mathbf{P}$ ).

Notice that the Hamiltonian $H$ in (1.2) is different from that of Russo and Smereka [26]. This comes from an additional approximation, after the dipole approximation, that they needed in their analysis and that we have removed. Their Hamiltonian is just the first order term in a Taylor expansion of $H$ in the parameter $\lambda$

$$
\begin{gathered}
H_{R S}=\frac{1}{2}|p|^{2}+p \cdot \nabla_{x} \Phi^{0}, \\
\Phi^{0}(t, x)=\lambda B * \mathbf{P} .
\end{gathered}
$$

Another advantage, compared to the system of [26], is that our system (1.1)(1.3) has a conserved energy (inherited from the Hamiltonian structure) which has the right form $\mathcal{E}_{T}=\mathcal{E}_{K}+\mathcal{E}_{P}$ (total energy = kinetic energy + potential energy) : 
Theorem 3.3 ([19]). Additionaly to the assumption of Theorem 3.1, assume that $B=D^{2} b$ for some function $b$. Then, the vector potential $\Phi$ satisfies $\Phi=\nabla \varphi$ and is given by the equation

$$
\varphi(t, x)=\lambda b * \operatorname{div}(\mathbf{P}+\rho \nabla \varphi)(t, .) .
$$

Then, the conserved energy can be written

$$
\mathcal{E}_{T}(t)=\int_{\mathbb{R}^{6}} H(t, x, p) g_{N} d x d p+\frac{1}{2 \lambda} \int_{\mathbb{R}^{3}}\left|\nabla_{x} \varphi(t, x)\right|^{2} d x .
$$

The structure of the equation (1.1)-(1.3) is mathematically interesting because it is semi-kinetic (the advection velocity $H_{p}$ depends on the parameter $p$ and a macroscopic quantity). Such a structure was also found for the kinetic formulation of isentropic gas dynamics in P.L. Lions, B. Perthame and E. Tadmor [23].Other examples can be found also in H. Spohn [31], C. Cercignani [9]. However, the singularity arising in the Hamiltonian, more precisely in the coeficient $H_{x}$ in the equation (1.1), is too strong to hope for an existence theory. In the Appendix we also show some numerical simulations which indicate that the short range interaction might be more important that comparing the collision rate for classical collisions $\mathrm{Na}^{2}$ to our kinetic parameter $\lambda \approx N a^{3}$.

Poof of Theorem 3.2. We indicate some steps toward the obtention of the Mean Field Equation for $g_{N}$. The first step is to write the Lagrangian dynamic equations (3.1) as

$$
\begin{gathered}
\left\{\begin{array}{c}
\frac{d}{d t} X_{i}(t)=V_{i}(t), \\
\frac{d}{d t} V_{i}=\lambda \partial_{t}\left[B \star j_{N}(t, .)\right]\left(X_{i}(t)\right),
\end{array}\right. \\
j_{N}(t, x)=\int_{\mathbb{R}^{3}} v f_{N}(t, x, v) d v=\frac{1}{N} \sum_{i=1}^{N} \delta\left(x-X_{i}(t)\right) .
\end{gathered}
$$

And this is because

$$
\begin{aligned}
\frac{d}{d t}\left(V_{i}(t)\right. & \left.-a^{3} \tau \sum_{j=1}^{N} B\left(X_{i}(t)-X_{j}(t)\right) V_{j}(t)\right) \\
& =-\frac{a^{3} \tau}{2} \sum_{k, l=1}^{N} V_{k}(t)^{t} \nabla_{x_{i}}\left[B\left(X_{k}(t)-X_{l}(t)\right)\right] V_{l}(t) .
\end{aligned}
$$

The second step is to deduce from (3.10) the equation on $f_{N}$. We have

$$
\begin{aligned}
& \frac{\partial f_{N}}{\partial t}+v \cdot \nabla_{x} f_{N}+F_{N} \cdot \nabla_{v} f_{N}=0, \\
& F_{N}(t, x)=\lambda\left(B * \partial_{t} j_{N}(t, .)\right)(x) .
\end{aligned}
$$


The final step is to change variables in this equation. We set, see (3.7),

$$
v=p+\Phi_{N}(t, x)=\nabla_{p} H(t, x, p)
$$

and we notice that

$$
\Phi_{N}(t, x)=\lambda B \star j_{N} .
$$

This simple change of variables in the system (3.11)- (3.12) yields the system (1.1)-(1.3) for

$$
g_{N}(t, x, p)=f_{N}(t, x, v)
$$

\section{Interaction of particles in a Stokes flow}

We adopt the same plan as before for describing the motion of spherical particles in a Stokes flow. We derive the dynamical system under the assumption that the particles are dilute enough to be described by a dipole approximation. In this Section, we explain this approximation and, in the next Section, we derive a class of kinetic equations for the evolution of their density in the phase space (space and velocity here) and we exhibit the relevant kinetic parameters. Notice that, here, we restrict ourselves to the simplest situation of a cloud of particles. Systems with a quasi uniform repartition at infinity have also been considered from a physical point of view (see [12] and the references therein).

\section{1 notations}

We now consider the case of $N$ balls interacting in a Stokes flow. Again, we use the notations of Section 2 but, here, new quantities are needed. We denote the kinetic momentum of the particles by $j_{p}=C \rho_{p} a^{5}$, their angular velocity $\Omega_{i}$. Also, we denote $\sigma(t, x)$ the stress tensor of the fluid flow aroung the particles. It is given for $1 \leq \alpha, \beta \leq 3$, by

$$
\sigma_{\alpha \beta}=-p \delta_{\alpha \beta}+\eta\left(\frac{\partial}{\partial x_{\alpha}} v_{\beta}+\frac{\partial}{\partial x_{\beta}} v_{\alpha}\right)
$$


where $v(t, x)$ denotes the fluid velocity. We finally assume that the fluid is now described by the Stokes system

$$
\left\{\begin{array}{l}
\eta \triangle v=\nabla p \text { in } \mathbb{R}^{3}-\bigcup_{\mathrm{i}=1}^{\mathrm{N}} \mathrm{B}_{\mathrm{i}}(\mathrm{t}) \\
\operatorname{div} v=0 \text { in } \mathbb{R}^{3}-\bigcup_{\mathrm{i}=1}^{\mathrm{N}} \mathrm{B}_{\mathrm{i}}(\mathrm{t}) \\
v=V_{i}+\Omega_{i} \wedge\left(x-X_{i}\right) \text { on } \partial B_{i}(t) .
\end{array}\right.
$$

Also, we assume that the fluid is at rest at infinity $v_{\infty}=0$. Then, the dynamics of the balls is defined by the fundamental principle of dynamics

$$
\left\{\begin{array}{l}
\dot{X}_{i}=V_{i} \\
m_{p} \dot{V}_{i}=F_{i}=-\rho_{f} \int_{\partial B_{i}(t)}(\sigma \cdot n) d S+\left(m_{p}-m_{f}\right) g, \\
j_{p} \dot{\Omega}_{i}=\Gamma_{i}=-\rho_{f} \int_{\partial B_{i}(t)}\left(x-X_{i}\right) \wedge(\sigma \cdot n) d S .
\end{array}\right.
$$

Where we have defined

$$
m_{f}=\frac{4 \pi}{3} a^{3} \rho_{f} .
$$

Notice that, again, the forces depend linearly on $\left(V_{i}, \Omega_{i}\right)_{1 \leq i \leq N}$. But again the related matrix depends on the particles positions in a very complex way. Also, we have denoted by $g$ the constant gravity vector.

Remark 4.1 It is useful to notice that, for $g=0$, there is a non-increasing energy. Since we neglected the fluid inertia, it is reduced to the kinetic energy of the particles

$$
\mathcal{E}(t)=\frac{1}{2} \sum_{i=1}^{N}\left(m_{p}\left|V_{i}\right|^{2}+j_{p}\left|\Omega_{i}\right|^{2}\right) .
$$

We can indeed compute

$$
\begin{aligned}
\frac{d}{d t} \mathcal{E}(t) & =\sum_{i=1}^{N} V_{i} \cdot F_{i}+\Omega_{i} \cdot \Gamma_{i} \\
& =\rho_{f} \sum_{i=1}^{N} \int_{\partial B_{i}(t)}\left[V_{i} \cdot(\sigma \cdot n)+\Omega_{i} \cdot\left(\left(x-X_{i}\right) \wedge(\sigma \cdot n)\right)\right] d S \\
& =\rho_{f} \sum_{i=1}^{N} \int_{\partial B_{i}(t)}\left(V_{i}+\Omega_{i} \wedge\left(x-X_{i}\right)\right) \cdot(\sigma \cdot n) d S,
\end{aligned}
$$

and therefore

$$
\begin{aligned}
\frac{d}{d t} \mathcal{E}(t) & =\rho_{f} \sum_{i=1}^{N} \int_{\partial B_{i}(t)} v \cdot(\sigma \cdot n) d S \\
& =-\rho_{f} \sum_{\alpha, \beta=1}^{3} \int_{\mathbb{R}^{3}-\cup \mathrm{B}_{\mathrm{i}}(\mathrm{t})}\left(\partial_{\alpha} v_{\beta}\right) \cdot\left(-p \delta_{\alpha \beta}+\eta\left(\partial_{\alpha} v_{\beta}+\partial_{\beta} v_{\alpha}\right)\right) d S \\
& =-\frac{\eta}{2} \rho_{f} \sum_{\alpha, \beta=1}^{3} \int_{\mathbb{R}^{3}-\cup \mathrm{B}_{\mathrm{i}}(\mathrm{t})}\left(\partial_{\alpha} v_{\beta}+\partial_{\beta} v_{\alpha}\right)^{2} d S \\
& =-\frac{\eta}{2} \rho_{f} \int_{\mathbb{R}^{3}-\cup \mathrm{B}_{\mathrm{i}}(\mathrm{t})}\left|\nabla v+(\nabla v)^{T}\right|^{2} d S \\
& \leq 0 .
\end{aligned}
$$




\section{2 case of a single bubble and Stokeslets}

The exact solution to the Stokes system for a single ball is known. To simplify the notations, we assume it is centered at $X_{1}=0$, and we set $V_{1}=V, \Omega_{1}=\Omega$.

In this situation, one readily checks the formulae

$$
\begin{gathered}
v=a\left(\frac{V}{|x|}+\frac{a^{2}-|x|^{2}}{4} \nabla \frac{V \cdot x}{|x|^{3}}\right)+\frac{a^{3}}{|x|^{3}} \Omega \wedge x, \\
p=-\frac{3}{4} a \eta V \cdot \frac{x}{|x|^{3}}, \\
\sigma=\quad \frac{3}{2} \frac{a}{|x|^{3}} \eta\left(-V \otimes x-x \otimes V+\frac{5 a^{2}-3|x|^{2}}{|x|^{4}} V \cdot x x \otimes x\right. \\
\left.+\frac{a^{2}-|x|^{2}}{|x|^{2}} V \cdot x I d\right)-3 \frac{a^{3}}{|x|^{5}}(x \otimes(\Omega \wedge x)+(\Omega \wedge x) \otimes x) .
\end{gathered}
$$

Using these expressions, we can compute the forces applied on the particle. After simple calculations and using the symmetries, we obtain, for $g=0$ to simplify,

$$
\begin{aligned}
& F=-6 \pi a \eta \rho_{f} V, \\
& \Gamma=-8 \pi a^{2} \eta \rho_{f} \Omega .
\end{aligned}
$$

Consequently, the motion of a single ball is reduced to simple friction with a decoupling of rotation and translation. Notice also that, combining these expression with the dynamics (4.2), we obtain

$$
\begin{aligned}
\dot{V} & =-\frac{9}{2} \eta \frac{\rho_{f}}{\rho_{p}} a^{-2} V, \\
\dot{\Omega} & =-C \eta \frac{\rho_{f}}{\rho_{p}} a^{-2} \Omega .
\end{aligned}
$$

Hence, the relaxation time for friction is of the same order for both velocity and momentum.

Eventually, at long distance (for large $x$ ), the main contribution to the velocity field in (4.5) is given by

$$
\begin{gathered}
\tilde{v}(x)=\frac{3}{4} a\left(\frac{V}{|x|}+\frac{V \cdot x}{|x|^{3}} x\right) . \\
\tilde{p}(x)=-\frac{3}{4} a \eta V \cdot \frac{x}{|x|^{3}} .
\end{gathered}
$$


This is the exact solution to the Stokes system with a point force at the origin proportional to $V$, namely

$$
\left\{\begin{array}{l}
\eta \Delta v_{i}^{1}=\nabla p_{i}^{1}-F_{0} \delta(x) \quad \text { in } \mathbb{R}^{3} \\
\operatorname{div} v_{i}^{1}=0 \quad \text { in } \mathbb{R}^{3}
\end{array}\right.
$$

This particular solution in $(4.10),(4.11)$ is called a Stokeslet.

\section{3 the method of reflections}

We can follow the same lines as in the potential case and build the solution to the N-particles Stokes system along the method of reflections. We expand the solution as a series expansion which aims to simplify the geometry of the domain and reduce it to the exterior of single balls.

$$
\begin{aligned}
& v(t, x)=\sum_{n=1}^{\infty} \sum_{i=1}^{N} v_{i}^{(n)}(t, x), \\
& p(t, x)=\sum_{n=1}^{\infty} \sum_{i=1}^{N} p_{i}^{(n)}(t, x) .
\end{aligned}
$$

Again, we use for the first term the single ball approximation

$$
\left\{\begin{array}{l}
\eta \Delta v_{i}^{(1)}=\nabla p_{i}^{(1)} \quad \text { in } \mathbb{R}^{3}-\mathrm{B}_{\mathrm{i}}(\mathrm{t}) \\
\operatorname{div} v_{i}^{(1)}=0 \quad \text { in } \mathbb{R}^{3}-\mathrm{B}_{\mathrm{i}}(\mathrm{t}) \\
v_{i}^{(1)}=V_{i}+\Omega_{i} \wedge\left(x-X_{i}\right) \quad \text { on } \partial B_{i}(t)
\end{array}\right.
$$

Then, we correct the boundary values thanks to the recursion formulae

$$
\left\{\begin{array}{c}
\eta \Delta v_{i}^{(n)}=\nabla p_{i}^{(n)} \quad \text { in } \mathbb{R}^{3}-\mathrm{B}_{\mathrm{i}}(\mathrm{t}) \\
\operatorname{div} v_{i}^{(n)}=0 \quad \text { in } \mathbb{R}^{3}-\mathrm{B}_{\mathrm{i}}(\mathrm{t}) \\
v_{i}^{(n)}=\sum_{j \neq i} v_{j}^{(n-1)} \quad \text { on } \partial B_{i}(t)
\end{array}\right.
$$

Summing up these equations, we see that the expressions in (4.13) formally satisfy the Stokes system (4.1) whenever the series converges, which again requires certainly an assumption on the distance between particles.

\section{4 the dipole approximation}

Like in the case of a potential flow, the first terms $v_{i}^{(1)}$ cannot be used alone; from the condition div $\sigma_{i}^{(1)}=0$, its contribution to the other balls $B_{j}$ vanishes 
and the motion is thus reduced to pure independent friction of the $\mathrm{N}$ particles as in the case of a single ball.

Therefore, we are lead to use the second term. This requires to input $v_{i}^{(1)}$ as a boundary condition for $v_{i}^{(2)}$, which we know explicitely from (4.5). And, again, assuming that the inter-particle distance $d_{i j} / a$ is large, we can approximate the boundary term on $\partial B_{i}(t)$ in $(4.15)$, as

$$
v_{i}^{(2)}=\sum_{j \neq i}\left[\frac{3 a}{4\left|X_{j}-X_{i}\right|} V_{j}+\frac{3 a}{4} V_{j} \cdot\left(X_{j}-X_{i}\right) \frac{X_{j}-X_{i}}{\left|X_{j}-X_{i}\right|}+O\left(\frac{a^{2}}{\left|X_{j}-X_{i}\right|^{2}}\right)\right]
$$

Neglecting the ' $O$ ' term in this equality, the velocity is constant on $\partial B_{i}(t)$. Hence, we can use again the single ball formulae for the forces. We obtain

$$
\begin{gathered}
\frac{1}{a \eta \rho_{f}} F_{i}=-6 \pi V_{i}-\frac{9}{2} \pi \sum_{j \neq i} \frac{a}{\left|X_{j}-X_{i}\right|}\left[V_{j}+V_{j} \cdot\left(X_{j}-X_{i}\right) \frac{X_{j}-X_{i}}{\left|X_{j}-X_{i}\right|^{2}}\right] \\
+\frac{\rho_{p}-\rho_{f}}{\eta a \rho_{f}} \frac{4}{3} \pi a^{3} g \\
\Gamma_{i}=-8 \pi a^{2} \eta \rho_{f} \Omega_{i} .
\end{gathered}
$$

As a consequence, in this dipole approximation, the rotation motion is still trivial. It is completely decoupled from the translational motion and reduced to simple friction. On the other hand, the translational motion undergoes a non-trivial interaction which is the sum of two-body interactions. It is therefore natural to neglect the momentum $\Omega$ in the sequel and to focus on this interaction.

The influence on $B_{i}(t)$ of the other balls results here in the sum of velocity terms of the form $\frac{a}{\left|X_{j}-X_{i}\right|}\left[V_{j}+V_{j} \cdot\left(X_{j}-X_{i}\right) \frac{X_{j}-X_{i}}{\left|X_{j}-X_{i}\right|^{2}}\right]$. These velocities are the Stokeslets centered at $X_{j}$-see (4.10). This is not surprising since we saw that in the case of a single particle, the force on the particle is proportional to the velocity on its boundary and that at long distance the velocity field created by a ball is a Stokeslet.

Compared to the case of the potential flow, the dipole approximation is much simpler for Stokes flow. The reason is that, from the condition $\operatorname{div} \sigma_{j}^{(n)}=0$, the only influence on a particle $B_{i}(t)$ comes from the term $v_{i}^{(n)}$ (with same index $i$ ) in the expression for the force $F_{i}$-see (4.2). This is not so simple for the case of a potential flow. 


\section{Kinetic and macroscopic eq. for particles in a Stokes flow}

We can now derive a kinetic equation for the density of particles submitted to the dynamics (4.2) and consider related mathematical problems. We explain this in a more general setting.

\section{1 the general interaction model}

From the discussion in the previous Section, we now consider a system of $\mathrm{N}$ particles interacting through a dynamic motivated by the dipole approximation of forces created by a Stokes flow. The general system we consider is therefore, for $1 \leq i \leq N$,

$$
\left\{\begin{array}{l}
\dot{X}_{i}(t)=V_{i}(t) \\
\dot{V}_{i}(t)=\lambda\left[-V_{i}(t)+\mu \frac{1}{N} \sum_{j \neq i} A\left(X_{j}-X_{i}\right) \cdot V_{j}+\kappa g\right] .
\end{array}\right.
$$

In the particular case of the Stokes flow we obtain the following values of the parameters real parameters $\lambda, \mu$,

$$
\begin{gathered}
\lambda=\frac{9}{2} \frac{\eta \rho_{f}}{a^{2} \rho_{p}}, \\
\mu=\frac{3}{4} a^{2} N, \\
\kappa=\frac{1}{\lambda} \frac{\rho_{p}-\rho_{f}}{\rho_{f}},
\end{gathered}
$$

and the $3 \times 3$ matrix $A$ is given by

$$
A(x)=-\frac{1}{|x|}\left[I d+\frac{x \otimes x}{|x|^{2}}\right] .
$$

This symmetric matrix has remarkable properties deduced from the fact that it is the fundamental solution of the Stokes equation.

Lemma 5.1 The matrix in (5.1) satisfies

$$
\operatorname{div} A=0,
$$

and it defines a non-positive operator,

$$
\int_{\mathbb{R}^{3}} u(x) A * u(x) d x \leq 0 \quad \text { for } u \neq 0 .
$$


Proof. The first property follows from an easy calculation. The second one can be made more precise. Being given a function $u(x) \in \mathbb{R}^{3}$, let us solve (coordinate by coordinate)

$$
-\Delta U=u, \quad \text { in } \mathbb{R}^{3} .
$$

Then, we have

$$
\int_{\mathbb{R}^{3}} u(x) A * u(x) d x=-\sum_{i=1}^{3} \int_{\mathbb{R}^{3}}\left|\nabla U_{i}\right|^{2} d x+\int_{\mathbb{R}^{3}}|\nabla \operatorname{div} U|^{2} d x \leq 0 .
$$

Also, notice that the singularity of the matix $A$ is not too bad, as it is

$$
A(x) \approx \frac{1}{|x|^{d-2}},
$$

(here we have always worked in three dimensions) and thus it is better than that of the classical Vlasov Poisson system where the singularity is $\approx \frac{1}{|x|^{d-1}}$. But the difficulty here is the bad dependency of the interaction term on $V_{j}$ which does not appear in the Vlasov case. Also, as in the Vlasov case, it is an open problem to prove a rigorous global limit as $N \rightarrow \infty$, even though the intuitive result is very simple.

A first step in this direction is to define

$$
f_{N}(t, x, p)=\frac{1}{N} \sum_{i=1}^{N} \delta\left(x-X_{i}(t)\right) \otimes \delta\left(v-V_{i}(t)\right) .
$$

One can readily check the following lemma

Lemma 5.2 The dynamic (5.1) is equivalent to the Vlasov-type equation (1.5), in the distributional sense, on the probability measure $f_{N}$ whenever the matrix $A$ is lipschitz continuous and bounded.

Since the matrix $A$ is the fundamental solution to Sokes equation, we are able to rewrite equation (1.5)

$$
\left\{\begin{array}{l}
\frac{\partial}{\partial t} f+v \cdot \operatorname{grad}_{x} f+\lambda_{1} \operatorname{div}_{v}((u-v) f)=0 \\
\Delta u(t, x)=\nabla p+\lambda_{2} j \\
j(t, x)=\int_{\mathbb{R}^{3}} v f(t, x, v) d v
\end{array}\right.
$$


This system looks very much like a model used by K. Hamdache (see [18]) and other authors which consists in replacing the second equation in the previous system by

$$
\begin{aligned}
& \partial_{t} u(t, x)-\eta \Delta u=\nabla p+\lambda_{2}(\rho u-j), \\
& \rho(t, x)=\int_{\mathbb{R}^{3}} f(t, x, v) d v .
\end{aligned}
$$

Except for the evolution term $\partial_{t} u$, the main difference with our equation is the change of sign for $\lambda_{2} j$ and the non-linear term $\lambda_{2} \rho u$ which is supposed to represent the full interaction between particles. Although it is more realistic, this model is also more complicated and in the following we will only deal with the more simple system (5.5) and usually under the form given by equation $(1.5)$.

\section{2 energy and long time behavior for the kinetic equa- tion}

From the energy property of the $N$-particles system - see also the calculation in the Remark 4.1 - we can hope an energy inequality for the kinetic system (1.5). We define the kinetic energy

$$
\mathcal{E}_{K}(t)=\frac{1}{2} \int_{\mathbb{R}^{6}}|v|^{2} f(t, x, v) d v d x,
$$

then, we have indeed,

$$
\begin{aligned}
\frac{d}{d t} \mathcal{E}_{K}(t) & =\lambda\left(-2 \mathcal{E}_{K}(t)+2 \mu \int_{\mathbb{R}^{3}} j A * j d x,\right. \\
& \leq-2 \lambda \mathcal{E}_{K}(t) .
\end{aligned}
$$

This last inequality is a simple consequence of the property (5.4). As a consequence, we deduce the dissipation rate of kinetic energy

$$
\mathcal{E}_{K}(t) \leq \mathcal{E}_{K}(0) e^{-2 \lambda t} .
$$

This property is fundamental in the theory developed in P.E. Jabin to prove the following result.

Theorem 5.3 [21] With the matrix A given by (5.1) and $g=0$, assume that the initial density $f(t=0)$ has finite energy and

$$
f(t=0) \in L^{1} \cap L^{\infty}\left(\mathbb{R}^{6}\right) .
$$


Then, the system (1.5) admits a weak solution and it satisfies for a.a. $t$, as $t \rightarrow \infty$

$$
f(t, x, v) \rightarrow \tilde{\rho}(x) \delta(v), \text { in } w-M^{1}\left(\mathbb{R}^{6}\right),
$$

for some density $\tilde{\rho}(x) \in L^{1} \cap L^{d /(d-2)}\left(\mathbb{R}^{3}\right)$.

One of the main difficulty in this theorem is the existence of a unique $\tilde{\rho}$ so that the full family converges (and not only subsequences). This relies on the precise energy dissipation rate. It is also proved in [21] that the total mass is conserved $\int_{\mathbb{R}^{3}} \tilde{\rho}(x) d x=\int_{\mathbb{R}^{6}} f(0, x, v) d x d v$.

\section{3 a macroscopic equation}

An interesting question arises from the size of $\lambda$ when a gravity term is present. Indeed, the above theory supposes $\lambda, \kappa$ and $\mu$ finite which is rather restrictive. On the other hand, since $a \rightarrow 0$ as $N \rightarrow \infty$ in order to keep $\mu$ finite, it is natural to assume that $\lambda \rightarrow \infty$. It is easy to see that after an appropriate rescalling of time and velocities, one can consider the limit $\lambda \rightarrow \infty$ keeping $\mu$ and $\kappa$ fixed.

We therefore consider the equation - in place of (1.5) -

$$
\begin{gathered}
\frac{\partial}{\partial t} f_{\lambda}+v \cdot \operatorname{grad}_{x} f_{\lambda}+\lambda \operatorname{div}_{v}\left(\left(A \star_{x} j-v+g\right) f_{\lambda}\right)=0 \\
j_{\lambda}(t, x)=\int_{\mathbb{R}^{3}} v f_{\lambda}(t, x, v) d v .
\end{gathered}
$$

When $\lambda \rightarrow \infty$, the formal limit of the density $f_{\lambda}$ in $(5.8)$ is

$$
f(t, x, v)=\rho(t, x) \delta(v-A \star j-g) .
$$

This gives a coupled system of nonlinear equations for $\rho$. The first equation is obtained in integrating the equation (1.5) in $v$, and we set $j=\rho u$,

$$
\left\{\begin{array}{l}
\frac{\partial}{\partial t} \rho+\operatorname{div}(\rho u(t, x))=0 \\
A *(\rho u)-u=g .
\end{array}\right.
$$

A more intuitive way to write this equation on $u$ is deduced from the fact that $A$ is the fundamental solution to the Stokes Equation.

$$
\left\{\begin{array}{l}
\operatorname{div} u=0 \\
\Delta u=\nabla p+\rho u \\
u(t, x) \rightarrow g, \quad \text { as }|x| \rightarrow \infty
\end{array}\right.
$$


From the free divergence condition, we deduce that the transport equation for $\rho$ shares the basic $L^{p}$ norm conservation property with the vorticity formulation of two dimensional Euler Equations for incompressible flows (see for instance J.Y. Chemin [11], C. Machioro and M. Pulvirenti [24]). A rigorous derivation of this limit $\lambda \rightarrow \infty$ is given in P.E. Jabin [22].

This kind of 'macroscopic' limit of a kinetic equation, without the help of a collision term is very exceptional compared to the classical relaxation toward a thermal equilibrium (see [9], [10]). There are other known cases of a similar phenomena. For instance, let us quote the gyrokinetic limit in plasma physics (see E. Frénot and E. Sonnendrucker [13]), the quasi-neutral limit of Vlasov-Poisson Equation (see Y. Brenier and E. Grenier [4], Y. Brenier [5]).

\section{Appendix 1. Numerical simulations in the case of a potential flow and short range ef- fect}

In this Section, we would like to report on some numerical simulations for a more complete problem of particles moving in a potential flow. This allows to address the question of short range effects and collisions which was left open in the sections 3 and 4. Additional results can be found in [6].

To the situation presented in Sections 2 and 3, we add the gravity forces and the friction term derived in Section 4. This allows more realistic computations. Also, we found it more convenient to work in the physical variables (velocity, not impulsion) and thus to consider the dynamic equation (3.10). This gives the equations of motion

$$
\begin{aligned}
\frac{d}{d t} X_{i}(t) & =V_{i}(t), \\
\frac{d}{d t} V_{i}(t) & =\gamma_{i}(t),
\end{aligned}
$$

where the acceleration is given by four different terms

$$
\begin{gathered}
\gamma_{i}(t)=\left(\gamma^{0}+\gamma^{1}+\gamma_{\eta}+\gamma_{g}\right)\left(X_{i}(t)\right), \text { with } \\
\gamma^{0}(x)=a^{3} \tau \sum_{1 \leq j \leq N} V_{j}(t)^{t} \nabla_{x} \underline{B}\left(x-X_{j}(t)\right) V_{j}(t), \\
\gamma^{1}(x)=a^{3} \tau \sum_{1 \leq j \leq N} \underline{B}\left(x-X_{j}(t)\right) \gamma^{0}\left(X_{j}(t)\right),
\end{gathered}
$$




$$
\begin{aligned}
\gamma_{\eta}(x) & =-\frac{12 \pi \eta a\left(V_{i}(t)-v_{f}(x)\right.}{\frac{4}{3} \pi a^{3}\left(\rho_{p}+\frac{\rho_{f}}{2}\right)}, \\
\gamma_{g}(x) & =\frac{\rho_{p}-\rho_{f}}{\rho_{p}+\rho_{f} / 2} g .
\end{aligned}
$$

The first two terms represent the added mass force (3.10) with a second order approximation in the kinetic parameter $\lambda$ in (2.1), which has the property to preserv the right energy structure (cf. [19], [6]). We recall the definition of $\underline{B}$ in (3.4). The term $\gamma_{\eta}$ represents the friction due to viscosity and the last term is the buoyant force (poussée d'Archimède). The fluid velocity is taken according to the potential gradient, deduced from Section 2,

$$
\begin{aligned}
v_{f}(x)=2 \pi a^{3} & \sum_{1 \leq j \leq N} \underline{B}\left(x-X_{j}(t)\right)\left[V_{j}(t)\right. \\
& \left.-2 \pi a^{3} \sum_{1 \leq k \leq N} \underline{B}\left(X_{j}(t)-X_{k}(t)\right) V_{k}(t)\right] .
\end{aligned}
$$

But a fundamental effect in this motion is that particles have a tendency to collide much more than expected from the classical rate of collision $n a^{2}$. This is due to the fact that particles moving with parallel velocities (this is frequent due to gravity) have a tendency to attract each other. Therefore it is fundamental to introduce collision rules. These have been taken as the usual hard-sphere collisions; postcollisional velocities are given by

$$
\begin{aligned}
V_{i}^{\prime} & =V_{i}-\left[\left(V_{i}-V_{j}\right) \cdot n\right] n \\
V_{j}^{\prime} & =V_{j}+\left[\left(V_{i}-V_{j}\right) \cdot n\right] n,
\end{aligned}
$$

where $n$ denotes the normal $n=\frac{x_{i}-x_{j}}{\left|x_{i}-x_{j}\right|}$. This is possible because we work directly in physical variables. In the impulsion variables this is also possible (see [32], [27])

We performed numerical tests In figures 1 and 2 we presente a three dimensional evolution of 125 bubbles, initially semiregularly distributed around a $5 \times 5 \times 5$ grid, with velocity zero.

The physical data are: diameter $=10^{-3} \mathrm{~m}$, concentration $=12.7 \%$ (this corresponds to a box which side is $8 \mathrm{~mm}$ long), gravity $=-9.81 e_{z}$, viscosity $=10^{-3} \mathrm{~Pa} . \mathrm{s}, \rho_{f}=1000 \mathrm{~kg} / \mathrm{m}^{3}, \rho_{p}=0$.

First, we observe the formation of horizontal continuous layers, normal to gravity (this is due to attraction of bubbles lifting in the same horizontal plane) next, a vertical repulsion between layers destabilize their shape. 
Finally we obtain, when bubbles reach their limit velocity (due to friction) a cloud of dispersed bubbles or pairs of bubbles (rather normal to gravity) elongated in the gravity direction. Especially, including the real collisions, we do not observe anymore the strong horizontal layering. This effect has been obtained with a modification of the short range forces to make them repulsive (see [19], [29], [30]). The main macroscopic effect rather comes from particles stiking together as can be seen in figures 1 and 2 (thick cicles are those touching each other in the three dimensional space).

Acknowledgment We would like to thank J.F. Bourgat and INRIA (project $\mathrm{M} 3 \mathrm{~N}$ ) for providing the numerical results in the Appendix, as well as B. Lucquin for a constant help in developing the numerical code. 

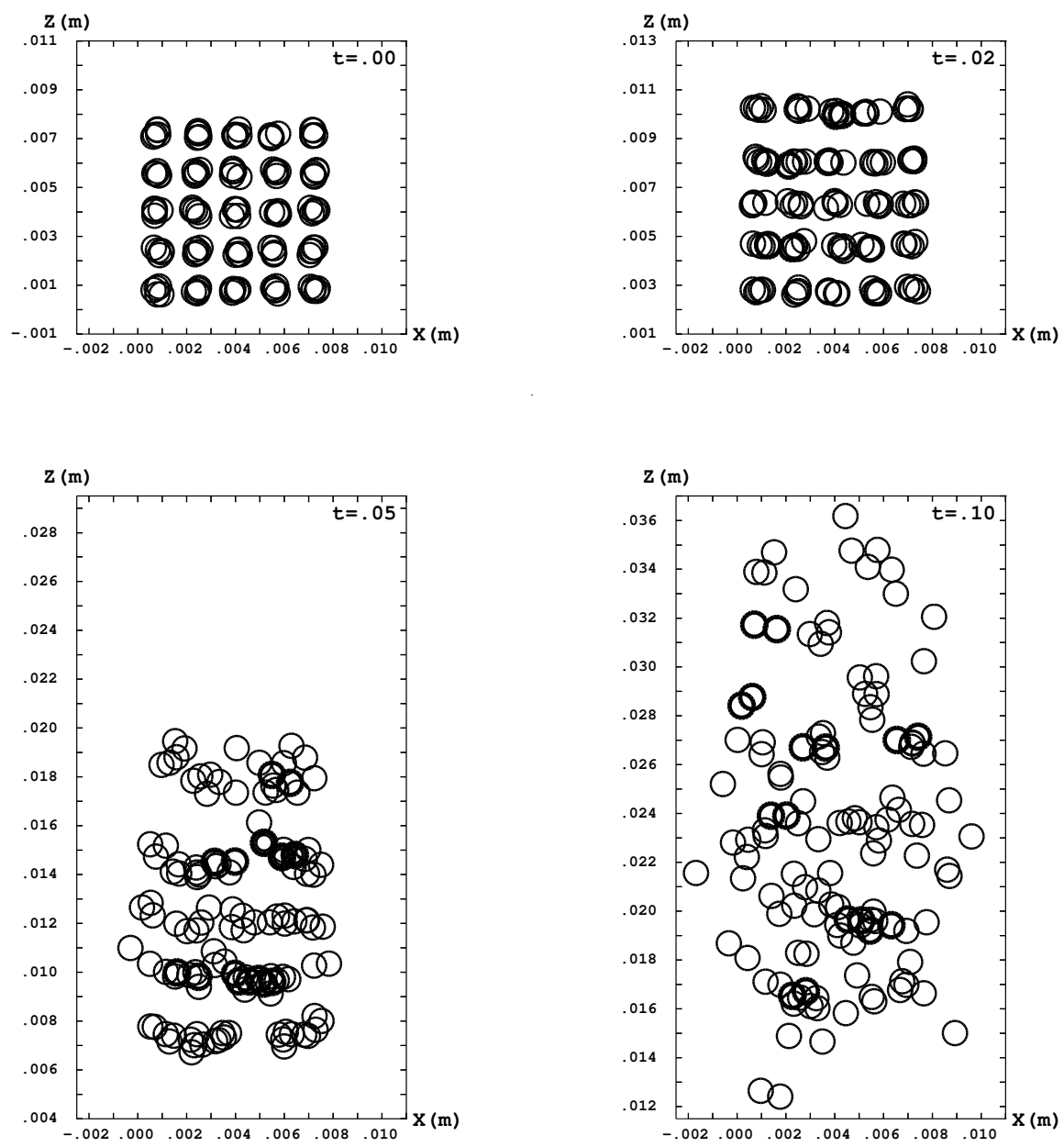

Figure 1: Snapshots of the evolution of 125 bubbles initially semiregularly distributed in a box, with velocity zero, at $\mathrm{t}=0 ., 0.02,0.05,0.1 \mathrm{~s}$. The circles are projections of bubbles on the vertical plane $\mathrm{xz}$ and thick cercles represent pairs or group of bubbles. 


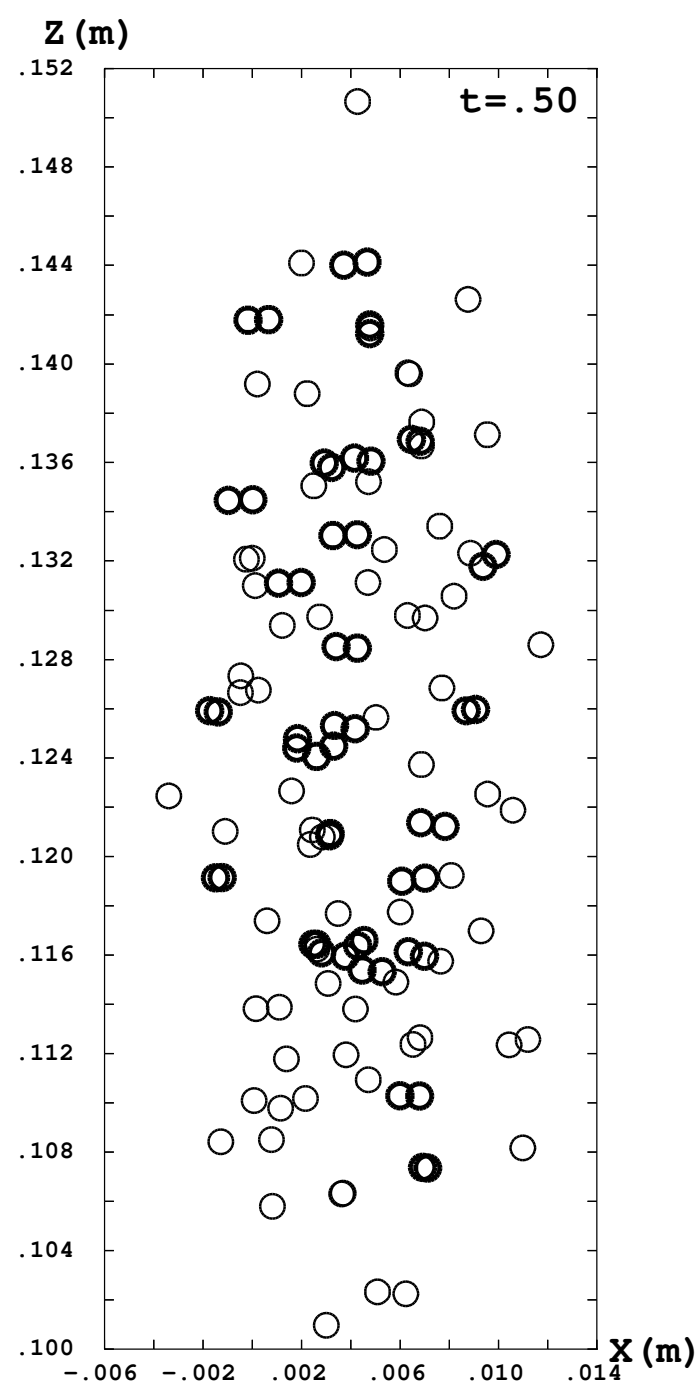

Figure 2: Distribution at $t=0.5 s$ of 125 bubbles. The circles are projections of bubbles on the vertical plane xz and thick circles represent pair of bubbles. 


\section{Appendix 2 : numerical simulations for a Stokes flow}

\subsection{Introduction}

We present here some numerical simulations for the simplified model of particles in a Stokes flow detailed in section 5.1 under the form of a kinetic equation for the dynamics of the particles. The purpose of this appendix is mainly to investigate numerically the long time behaviour of this equation in the case where there is gravity.

In the case without gravity, the result is known (see section 5.2 ) and the solution concentrates towards zero velocities. It should be noticed that equation 1.5 is not invariant under galilean transformations. This simply comes from our assumption that the fluid is at rest at infinity. Because of this lack of galilean invariance in the equation, we cannot reduce the case with gravity to the case without gravity.

However even with this remark, one does not expect a completely different behaviour for the cases with or without gravity. Hence a reasonable conjecture for the case with gravity could have been the following

Conjecture 1 The solution $f$ to equation 1.5 converges weakly to $\tilde{\rho} \delta\left(v-v_{0}\right)$ for some $\tilde{\rho}(x)$ and $v_{0}(x)$ depending on the parameters of the equation and possibly the initial data.

A weaker conjecture could also be as follows.

We define the functional

$$
F(t)=\int_{\mathbb{R}^{6}}\left|v-v_{m}\right|^{2} f(t, x, v) d x d v,
$$

where $v_{m}$ is the average velocity

$$
v_{m}(t)=\left(\int_{\mathbb{R}^{6}} v f(t, x, v) d x d v\right) /\left(\int_{\mathbb{R}^{6}} f d x d v\right) .
$$

Conjecture 2 The solution to equation 1.5 concentrates in velocity in long time. More precisely, $v_{m}$ tends to some $v_{0}$ and the functionnal $F$ just defined in ( 7.1 ) converges to zero as the time goes to infinity.

As surprising (or unsurprising) as it may seem, these two conjectures cannot be numerically verified at all. Based on numerical evidences, the asymptotic behaviour is thus completely different when we add a gravity. 


\subsection{Presentation of the computation}

We solve numerically the system

$$
\begin{aligned}
\frac{d}{d t} X_{i}(t) & =V_{i}(t) \\
\frac{d}{d t} V_{i}(t) & =-\lambda V_{i}(t)+\frac{1}{N} \sum_{j=1}^{N} A_{\eta}\left(X_{j}-X_{i}\right) \cdot V_{j}+g .
\end{aligned}
$$

This corresponds to the system 5.1 normalized with $\mu=\kappa=\frac{1}{\lambda}$ except that in the interaction term of 7.4 the sum is done for all indices $j$ including $i$. The matrix $A_{\eta}$ is a regularisation of the matrix $A$ defined in section 5.1, more precisely

$$
A_{\eta}(x)=-\frac{1}{|x|+\eta}\left(I d+\frac{x \otimes x}{(|x|+\eta)^{2}}\right) .
$$

In the simulations presented further, $\lambda$ and $\eta$ are chosen equal to $0.1, g$ is the vector $(0,0,1)$ and we take 200 particles.

Solving numerically the previous system presents no significant problem once you have chosen a time step small enough for stability. Moreover by opposition to the case of a potential flow, short range effects are not important here. In fact, although the interaction does not completely prevent them, collisions are very rare and usually the particles remain far enough from each other.

We have considered three kinds of initial conditions. The first one corresponds to random position in the cube $[0,1]^{3}$ and random velocities in $[-30,30]^{3}$. The second one consists in taking $X_{i}(0)=(i-1,0,0)$ and $V_{i}(0)=(0,0,1)$, and the last one $X_{i}(0)=(i-1,0,0)$ and $V_{i}(0)=(0,0, i-1)$.

\subsection{Conclusions}

The figures below clearly show that the velocity fluctuation $F(t)$ does not vanish. On other tests which we do not present here, we never observed a concentration of velocities.

Another computation, not shown here, concerns the minimal distance between particles. We indeed checked that collisions are extremely rare : particles never come close to each other. 
Acknowledgements. We did these simulations during a stay at the Erwin Schrodinger Institute in Vienna and we would like to thank the members of the Institute and especially P. Pietra for her help and advices. 


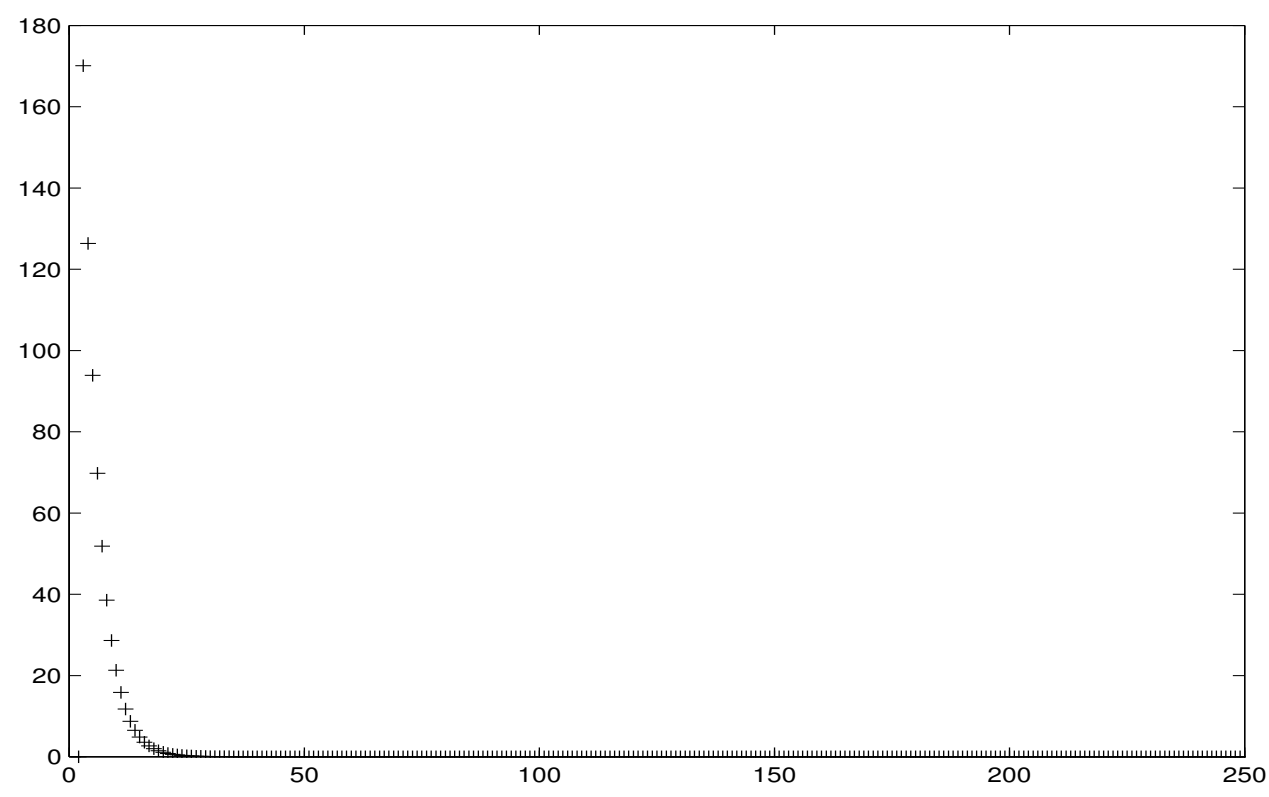

Figure 3: concentration in velocity $F(t)=\frac{1}{N} \sum_{i=1}^{N}\left|V_{i}(t)-V_{m}(t)\right|^{2}$, with $V_{m}=\frac{1}{N} \sum_{i=1}^{N} V_{i}(t)$, in the case of random initial positions and velocities. This seems to indicate that $F(t)$ converges towards zero but it is false as the next picture shows it. 


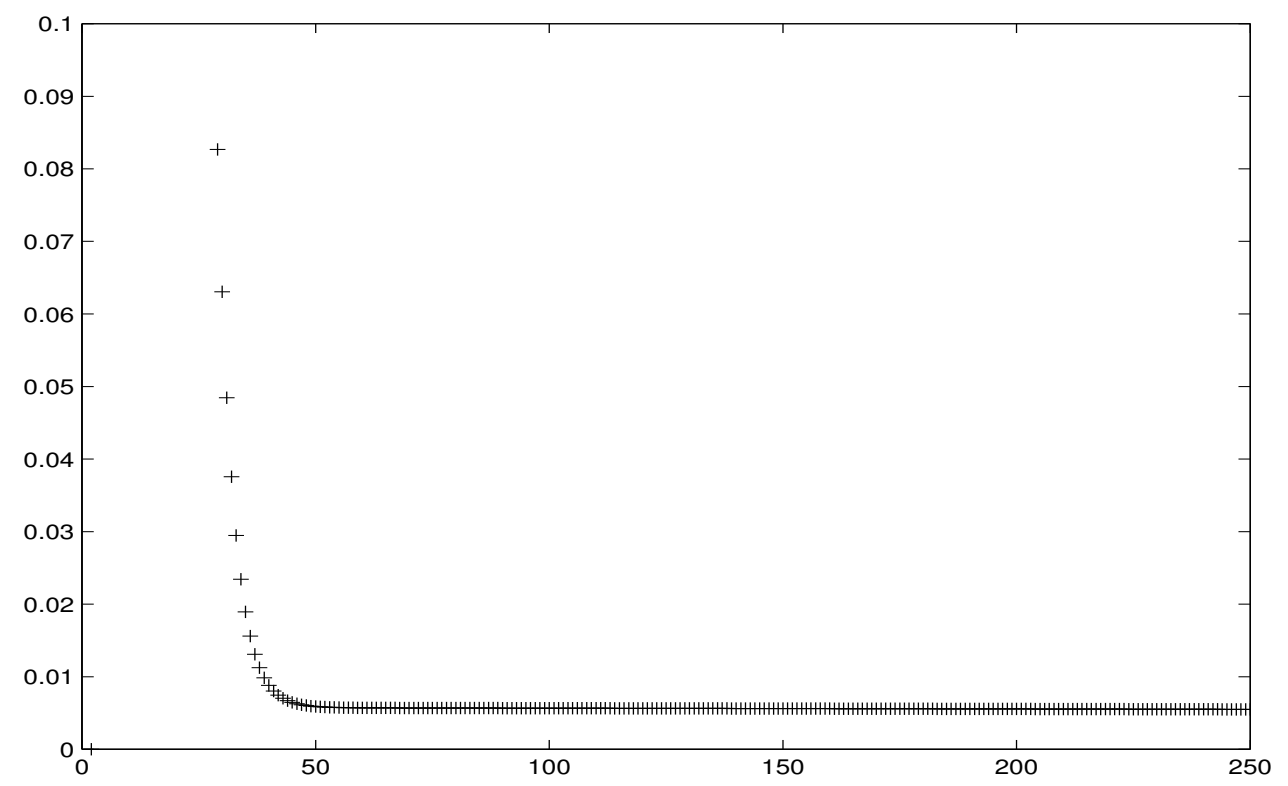

Figure 4: same as figure 1 but rescaled along the vertical axis. The functional $F(t)$ stops decreasing after a while. 


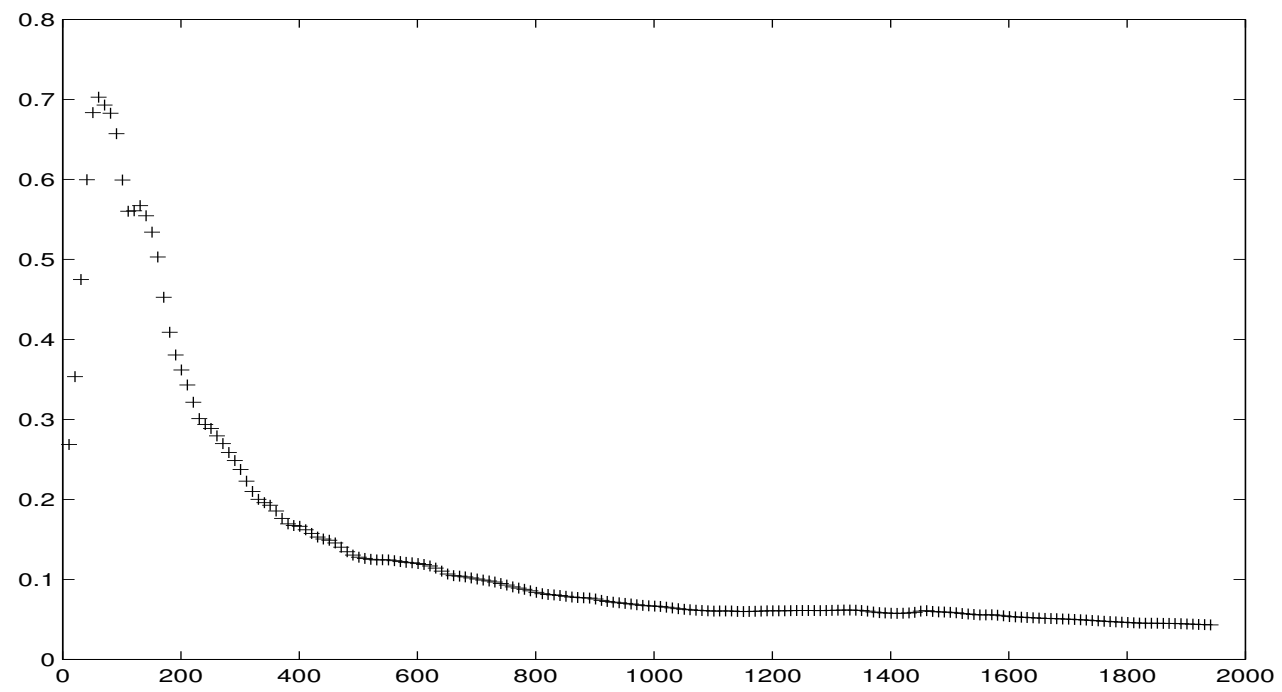

Figure 5: concentration in velocity $F(t)=\frac{1}{N} \sum_{i=1}^{N}\left|V_{i}(t)-V_{m}(t)\right|^{2}$, with $V_{m}=\frac{1}{N} \sum_{i=1}^{N} V_{i}(t)$, with $X_{i}(0)=(i-1,0,0)$ and $V_{i}(0)=(0,0,1)$. Again $F(t)$ does not converges towards zero. The stabilisation time and level are quite different from the previous picture, and therefore it is not due to a numerical artefact.

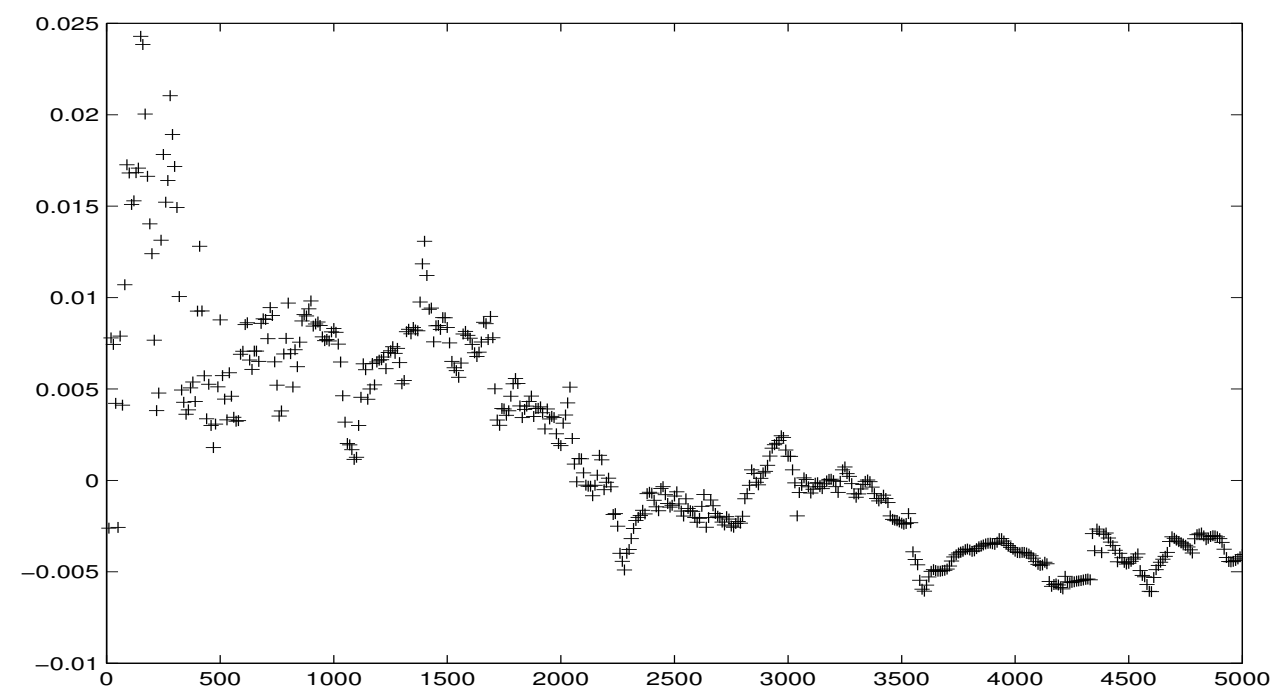

Figure 6: first coordinate of the average velocity $V_{m}$, with $X_{i}(0)=(i-1,0,0)$ and $V_{i}(0)=(0,0,1)$. The fluctuation never vanishes. 


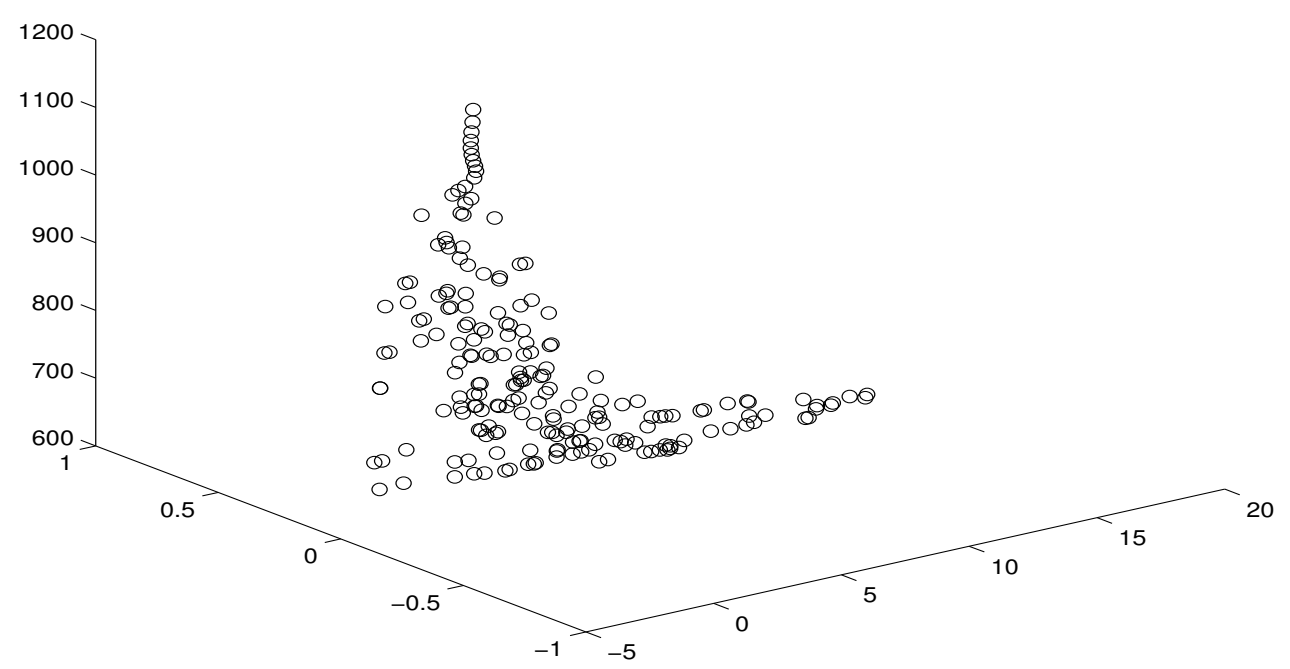

Figure 7: this picture shows the position of all the particles at time 200, with initial repartition $X_{i}(0)=(i-1,0,0)$ and $V_{i}(0)=(0,0, i-1)$. 


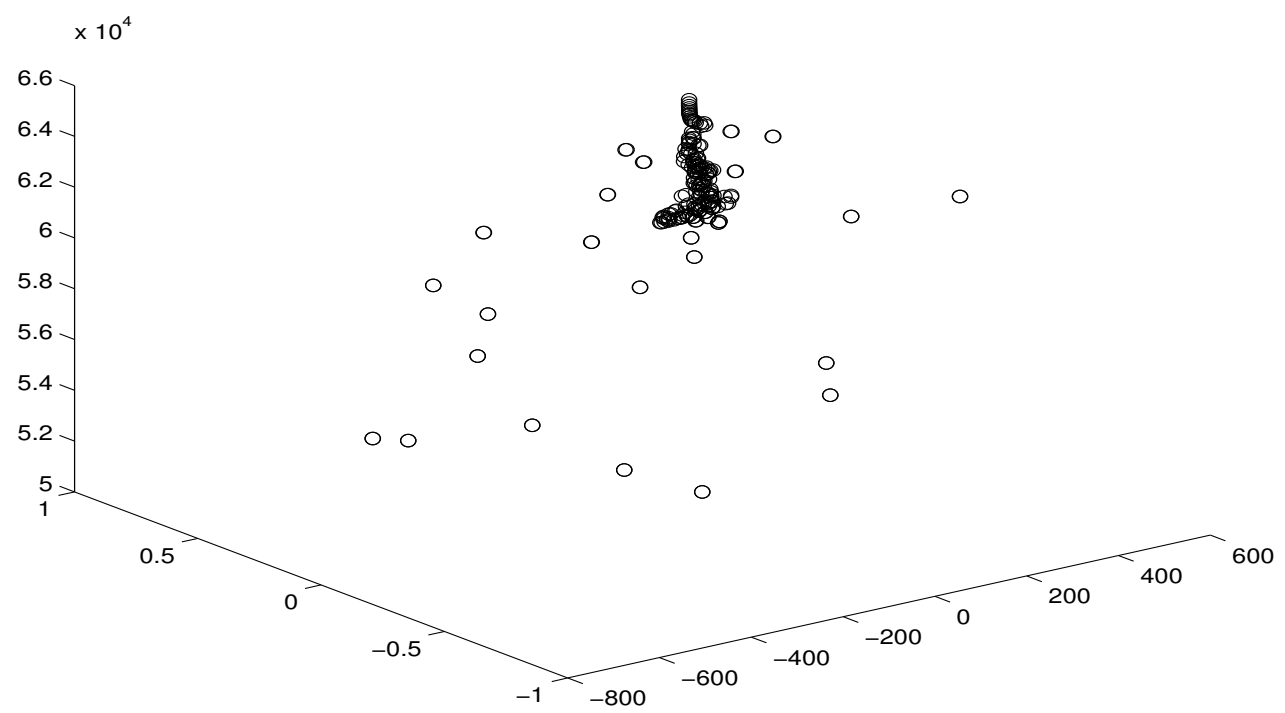

Figure 8: this picture shows the position of all the particles at time 10000, with initial repartition $X_{i}(0)=(i-1,0,0)$ and $V_{i}(0)=(0,0, i-1)$. 


\section{References}

[1] V.I. Arnold, Mathematical Methods of Classical Mechanics, Grad. Texts. Math. 60, Springer, (1978).

[2] G.K. Batchelor, An Introdution to Fluid Dynamics, Cambridge University Press, (1967).

[3] D. Benedetto, E. Caglioti and M. Pulvirenti, A kinetic equation for granular media, M2AN, Vol. 31(5) (1997), 615-642.

[4] Y. Brenier, E. Grenier, Limite singulière du système de Vlasov-Poisson dans le régime de quasineutralité: le cas indépendant du temps, C. R. Acad. Sc. Paris, t. 318, Série I (1994) $121-124$.

[5] Y. Brenier, Convergence of the Vlasov-Poisson system to the incompressible Euler equations, to appear in C.P.D.E.

[6] J.F. Bourgat, B. Lucquin-Desreux and B. Perthame, Motion of dispersed bubbles in a potential flow. 21st Int. Symp. on Rarefied Gas Dynamics. Marseilles, July 1998.

[7] H.F. Bulthuis, A. Prosperetti and A.S. Sangani, Particle stress in disperse two-phase potential flow, J. Fluid Mech., vol. 294 (1995) 1-16.

[8] G.K. Batchelor and C.S. Wen, Sedimentation in a dilute polydispersed system of interacting spheres, J. Fluid Mech. 124, (1982) 495-528.

[9] C. Cercignani, The Boltzmann equation and its application, Applied Math. Sciences 67, Springer-Verlag, Berlin (1988).

[10] C. Cercignani, R. Illner and M. Pulvirenti, The mathematical theory of dilute gases, Applied Math. Sciences 106, Springer-Verlag, Berlin (1994).

[11] J.Y. Chemin, Fluides parfaits incompressibles, Astérisque 230, S.M.F. (1995). 
[12] F. Feuillebois, Sedimentation in a dispersion with vertical inhomogeneities, J. Fluid Mech. 139, (1984) 145-172.

[13] E. Frénod And E. SONNENDRÜCKER, Long time behaviour of the two-dimensional Vlasov equation with a strong external magnetic field, INRIA report 3428, (1998).

[14] R.T. GLAssey, The Cauchy problem in kinetic theory, SIAM publications, Philadelphia (1996).

[15] R. Glowinski, T.W. Pan and J. PÉriaux, A fictitious domain method for external incompressible viscous flow modeled by Navier-Stokes equations, Comput. Meth. Mech. Engnr. 112, (1994), 133-148.

[16] E.J. Hinch, An averaged-equation approach to particle interactions in a fluid suspension, J. Fluid Mech. 83, (1977) 695-720.

[17] J. Happel And H. Brenner, Low Reynolds Number Hydrodynamics, Prentice-Hall, (1965).

[18] K. Hamdache, Global existence and large time behaviour of solutions for the Vlasov-Stokes equations, Japan J. Ind. and Appl. Math. To appear.

[19] H. Herrero, B. Lucquin-Desreux and B. Perthame, On the motion of dispersed bubbles in a potential flow -a kinetic description of the added mass effect, Publ. Lab. An. Num. Paris VI.

[20] R. Herczynski and I. Pienkowska, Towards a statistical theory of suspension, Ann. Rev. Fluid Mech. 12, (1980) 237-269.

[21] P.E. JABIN, Large time concentrations for solutions to kinetic equations with energy dissipation, Com. in P.D.E. To appear.

[22] P.E. Jabin, Doctoral Dissertation, Univ. Paris 6. In preparation.

[23] P.L. Lions, B. Perthame and E. Tadmor, Kinetic formulation of isentropic entropy solutions to isentropic gas dynamics system in Eulerian and Lagrangian variables, Comm. Math. Phys. 163 (1994) 415-431. 
[24] C. Marchioro and M. Pulvirenti, Vortex method in twodimensional fluid dynamics, Lecture Notes in Physics, 203, Springer (1984).

[25] B. Maury, R. Glowinski, Fluid particle flow: a symmetric formulation, C. R. Acad. Sci. Paris, t. 324, Série I, p. 1079-1084, 1997.

[26] G. Russo and P. Smereka, Kinetic theory for bubbly flow I: collisionless case, SIAM J. Appl. Math., Vol. 56, n²2 (1996), 327357.

[27] G. Russo and P. Smereka, Kinetic theory for bubbly flow II: fluid dynamic limit, SIAM J. Appl. Math., Vol. 56, n²2 (1996), 358-371.

[28] A. S. Sangani and A.K. Didwania, Dispersed-phase stress tensor in flows of bubbly liquids at large Reynolds numbers, $J$. Fluid Mech., vol. 248 (1993), 27-54.

[29] A. S. Sangani and A.K. Didwania, Dynamic simulations of flows of bubbly liquids at large Reynolds numbers, J. Fluid Mech., vol. 250 (1993), 307-337.

[30] P. Smereka, On the motion of bubbles in a periodic box, J. Fluid Mech. 254 (1993) 79-112.

[31] H. Spohn, Large scale dynamics of interacting particles, SpringerVerlag, Berlin, (1991).

[32] Y. Yurkovetsky, J.F. Brady, Statistical mechanics of bubbly liquids, Phys. Fluids 8 (4), (1996) 881-895. 
